\title{
Contactless prompt tumbling rebound of drops from a sublimating slope
}

\author{
Carlo Antonini, ${ }^{1, *}$ Stefan Jung, ${ }^{1}$ Andreas Wetzel, ${ }^{1}$ Emmanuel Heer, ${ }^{1}$ Philippe Schoch, ${ }^{1}$ \\ Ali Mazloomi Moqaddam, ${ }^{2}$ Shyam S. Chikatamarla, ${ }^{2}$ Ilya Karlin, ${ }^{2}$ Marco Marengo, ${ }^{3}$ \\ and Dimos Poulikakos ${ }^{1, \dagger}$ \\ ${ }^{1}$ Laboratory of Thermodynamics in Emerging Technologies, Mechanical and Process Engineering \\ Department, ETH Zurich, 8092 Zürich, Switzerland \\ ${ }^{2}$ Aerothermochemistry and Combustion Systems Laboratory, Mechanical and Process Engineering \\ Department, ETH Zurich, 8092 Zurich, Switzerland \\ ${ }^{3}$ School of Computing, Engineering and Mathematics, University of Brighton, Lewes Road, \\ Brighton BN2 4GJ, United Kingdom
}

(Received 12 January 2016; published 25 May 2016)

\begin{abstract}
We have uncovered a drop rebound regime, characteristic of highly viscous liquids impacting tilted sublimating surfaces. Here the drops, rather than showing a slide, spread, recoil, and rebound behavior, exhibit a prompt tumbling rebound. As a result, glycerol surprisingly rebounds faster than three orders of magnitude less viscous water. When a viscous drop impacts a sublimating surface, part of its initial linear momentum is converted into angular momentum: Lattice Boltzmann simulations confirmed that tumbling owes its appearance to the rapid transition of the internal angular velocity prior to rebound to a constant value, as in a tumbling solid body.
\end{abstract}

DOI: 10.1103/PhysRevFluids.1.013903

\section{INTRODUCTION}

Despite its illusory simplicity, the interaction between a liquid drop and a solid surface during impact is a fascinating fluidics problem, combining a variety of phenomena at multiple temporal and spatial scales [1-5]. These include splash [6-10], phase-change-induced surface levitation [11-15], skating on a film of trapped air [16-18], and rebounding [19-22]. Recently [11], it was demonstrated that drops can rebound after impact on an extremely cold solid carbon dioxide surface $\left(\right.$ at $-79{ }^{\circ} \mathrm{C}$, well below the limit of even homogeneous nucleation of water), because of the formation of a sublimated vapor layer acting both as impact cushion and thermal insulator, enabling drops to hover and rebound without freezing. A sublimating surface is different from aerodynamically assisted surface levitation [23-25] and from the Leidenfrost effect [12-14,26-28], in the sense that it is independent from liquid properties, such as boiling temperature, and there is no loss of drop mass due to its own boiling (as in the Leidenfrost phenomenon). Of course, in both cases an intervening layer is generated between the drop and the substrate. Sublimating surfaces can thus be used to study the contactless interaction of virtually any liquid, such as the highly viscous liquids used here. Also, they enable the study of phenomena expected from a superhydrophobic surface with extreme performance [29] (very high contact angles and very low hysteresis), providing further motivation for the fabrication and subsequent study of such surfaces. In the present fundamental study, we demonstrate and explain the existence of a prompt tumbling rebound mechanism, in which a small conversion of translational to rotational kinetic energy, at nonaxisymmetric impact conditions, promotes fast drop rebound despite high viscosities.

By focusing on nonaxisymmetric impact conditions at increasing viscosity, we observe a transition from an expected slide, spread, recoil, and rebound mechanism [see Figs. 1(a) and 1(b) and video 1

\footnotetext{
*Present address: EMPA, Swiss Federal Laboratories for Materials Science and Technology, Überlandstrasse 129, CH-8600 Dübendorf, Switzerland; Corresponding author: carlo.antonini@empa.ch

†Corresponding author: dpoulikakos@ethz.ch
} 

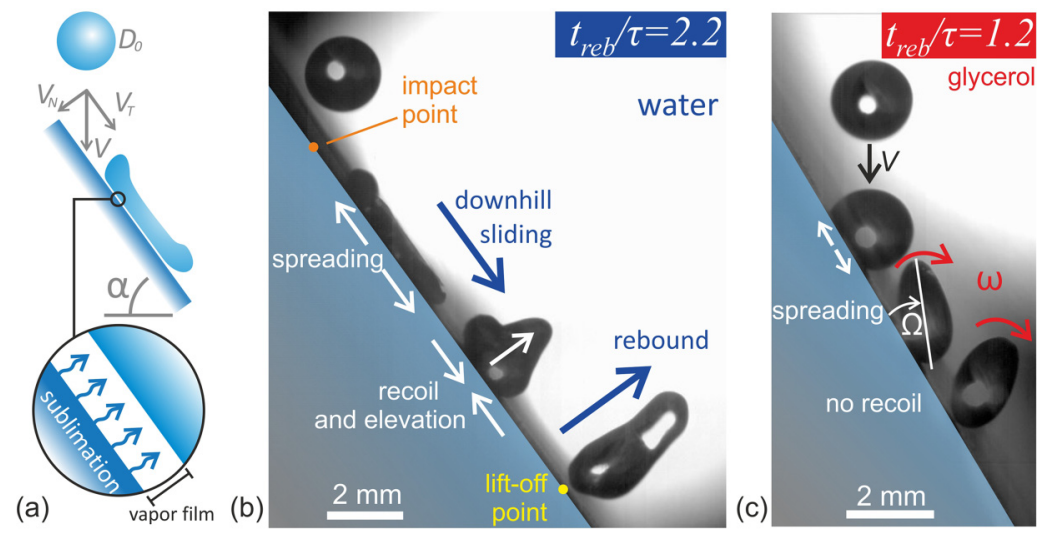

FIG. 1. Drop impact onto a tilted carbon dioxide sublimating surface: (a) schematic and image sequence of (b) water and (c) glycerol drop impacts. The impact conditions are $\alpha=55^{\circ}$, We $=\rho V^{2} D_{0} / \sigma=86$, and $\mathrm{Oh}=\mu / \sqrt{\rho \sigma D_{0}}=2.7 \times 10^{-3}$ for water and $\alpha=60^{\circ}, \mathrm{We}=121$, and $\mathrm{Oh}=3.2$ for glycerol. The image sequence was obtained by overlapping four images; see videos 1 and 2 in the SM [30] for the full sequence. The nondimensional rebound time is $t_{\mathrm{reb}} / \tau=2.2$ for water and $t_{\mathrm{reb}} / \tau=1.2$ for glycerol. Also indicated are the normal $V_{N}$ and tangential $V_{T}$ components of impact velocity, as well as the substrate tilt angle $\alpha$.

in the Supplemental Material [30]] to a tumbling behavior, enabling the prompt rebound of highly viscous liquids such as glycerol [see Fig. 1(c) and videos 2 and 3 in the SM [30]]. Here, after an initial viscosity-limited and impact-driven deformation phase, the drops simply tumble off the surface, rebounding faster than three orders of magnitude less viscous (water) drops. As confirmed by three-dimensional numerical simulations based on the entropic lattice Boltzmann method (ELBM) (see video 4 in the SM [30]), the behavior at high viscosities is aided by the contactless nature of the impact and was not observed on classical superhydrophobic surfaces, where an increase of viscosity can protract the rebound time [31] or even prevent rebound. In experiments on micropillar-based tilted superhydrophobic surfaces, we observed that viscous drops stay initially pinned at the impact point and eventually roll down the surface at a speed of approximately $10^{-2} \mathrm{~m} / \mathrm{s}$ (see Sec. SM1 in [30] for details on superhydrophobic surface preparation and video 5 in the SM [30] showing viscous drop behavior). In addition, drop pinning may occur on textured superhydrophobic surfaces as a result of drop impalement at high impact speed [22,31,32]: Impalement is not an issue for sublimating surfaces.

A representative schematic of a plausible scenario of contactless drop impact on a tilted sublimating surface is shown in Fig. 1(a): After impact, the drop slides down the slope and simultaneously spreads. The presence of the $\mathrm{CO}_{2}$ vapor layer due to substrate sublimation significantly reduces friction between the drop and the solid substrate, playing a role similar to that of the lubricating melted liquid water layer in ice skating [33]. The drop subsequently bounces off the surface after a certain rebound time $t_{\text {reb }}$, defined as the time lag between the impact and the lift-off. The corresponding downhill distance traveled by the drop $L_{\text {slide }}$ was measured from the impact point to the lift-off point [see Fig. 1(b)].

\section{METHOD AND MATERIALS}

\section{A. Experimental tests}

Drop impact studies were performed at room temperature $\left(\mathrm{T} \approx 23^{\circ} \mathrm{C}\right)$ on a carbon dioxide disk at $-79^{\circ} \mathrm{C}$, corresponding to the $\mathrm{CO}_{2}$ sublimation temperature at $1 \mathrm{~atm}$. The surface was first kept horizontal to study normal impacts as a basis for comparison and was subsequently tilted up to an angle of $75^{\circ}$ to study oblique impacts. Most of the experiments were conducted using water, glycerol, and water-glycerol mixtures, spanning over three orders of magnitude of viscosity (from $\sim 1$ to 
$\sim 10^{3} \mathrm{mPa}$ s), with surface tension $63<\sigma<72 \mathrm{mN} / \mathrm{m}$. Additional normal impact experiments were performed with glycols and silicon oils, with surface tension down to $20 \mathrm{mN} / \mathrm{m}$ (see details on liquid properties in Sec. SM2 in the SM [30]). The drop impact velocities and drop diameters were $0.8<$ $V<3.2 \mathrm{~m} / \mathrm{s}$ and $1.3<D_{0}<2.1 \mathrm{~mm}$, respectively. The corresponding nondimensional number ranges were for the Weber number $20<\mathrm{We}=\rho V^{2} D_{0} / \sigma<610$ or the Reynolds number $1.45<$ $\operatorname{Re}=\rho V D_{0} / \mu<5490$, and for the Ohnesorge number, $2.7 \times 10^{-3}<\mathrm{Oh}=\mu / \sqrt{\rho \sigma D_{0}}<4.92$.

\section{B. Numerical simulations}

To provide insight into the appearance of tumbling, we used the ELBM modeling approach [34], employing the Navier-Stokes equations for a two-phase fluid, where a van der Waals-type equation of state and Korteweg's stresses are implemented in the kinetic lattice Boltzmann setting of discrete velocity populations [35-37]. The impacting liquid was modeled as a drop on a superhydrophobic surface [38] with the contact angle $\theta=180^{\circ}$ and partial slip at the wall (see Ref. [39] for details on application of boundary conditions for the lattice Boltzmann populations at the wall). Partial slip at the wall was imposed using the slip coefficient $k$ obtained from the experimental measurements, presented below. The tangential velocity at the wall nodes was made equal to $k V_{t}^{\prime}$, where $V_{t}^{\prime}$ is the tangential velocity at the neighboring node within the fluid, in the direction perpendicular to wall, thus locally enforcing the partial slip observed in the experiments. This procedure helps us circumvent explicit modeling of the submicron gas layer trapped between the drop and the substrate. The validity of the numerical simulations is confirmed a posteriori from the good prediction of the rebound time, angular velocity, and drop shape, in comparison to the experiments.

\section{RESULTS AND DISCUSSION}

\section{A. Normal axisymmetric impacts}

The identification of a transition in the drop dynamics at high viscosity, i.e., high Oh, can be understood by first looking at the behavior of drops during normal impact on horizontal surfaces (Fig. 2) and then oblique impact on tilted surfaces (Figs. 3 and 4). Figure 2(a) illustrates the reboundno-rebound behavior on the We-Oh plane, for normal drop impact on a horizontal sublimating substrate. It is found that a transition from rebound to no-rebound occurs at $\mathrm{Oh} \approx 1$, with lowviscosity drops always rebounding for $\mathrm{Oh}<0.6$ and high-viscosity drops unable to lift off for $\mathrm{Oh}>1.2$. In the in-between transition regime, either outcome is possible. The transition regime at $\mathrm{Oh} \approx 1$ can be explained by the fact that in this range the viscous effects become of the same order as the surface energy effects and cause a rapid dissipation of the initial kinetic energy of the impacting drop: Viscous effects thus inhibit the conversion of kinetic energy into surface potential energy and back to kinetic energy, as typically occurs for relatively-low-viscosity liquids, such as water, and prohibit rebound. As shown in the inset in Fig. 2(b), at high Oh the highly viscous drops bead up to a quasispherical shape at rest and eventually roll away at the slightest perturbation, because of the absence of wetting and lateral adhesion forces due to the sublimating substrate. In the rebound regime, the rebound time follows the conventional scaling $t_{\text {reb }} / \tau=a=$ const, where $\tau=\left(\rho D_{0}^{3} / 8 \sigma\right)^{0.5}[19]$ and $a=2.2 \pm 0.2$ [20], with $a$ being constant and independent of the Ohnesorge number (see also more details in Sec. SM3 in the SM [30]).

\section{B. Oblique nonaxisymmetric impacts}

For nonaxisymmetric impacts on tilted sublimating surface, however, experiments reveal a qualitative change of the rebound pattern, showing that highly viscous glycerol drops can also rebound and do this even faster than water drops. The characteristic sliding velocity of the drop $V_{\text {slide }}$, computed as the ratio $L_{\text {slide }} / t_{\text {reb }}$, is plotted in Fig. 3(a) as a function of the tangential component of the impact velocity $V_{T}$. In the ideal inviscid case, assuming zero lateral adhesion forces [40,41] to cause drop deceleration and negligible acceleration due to gravity, the drop will continue to travel 


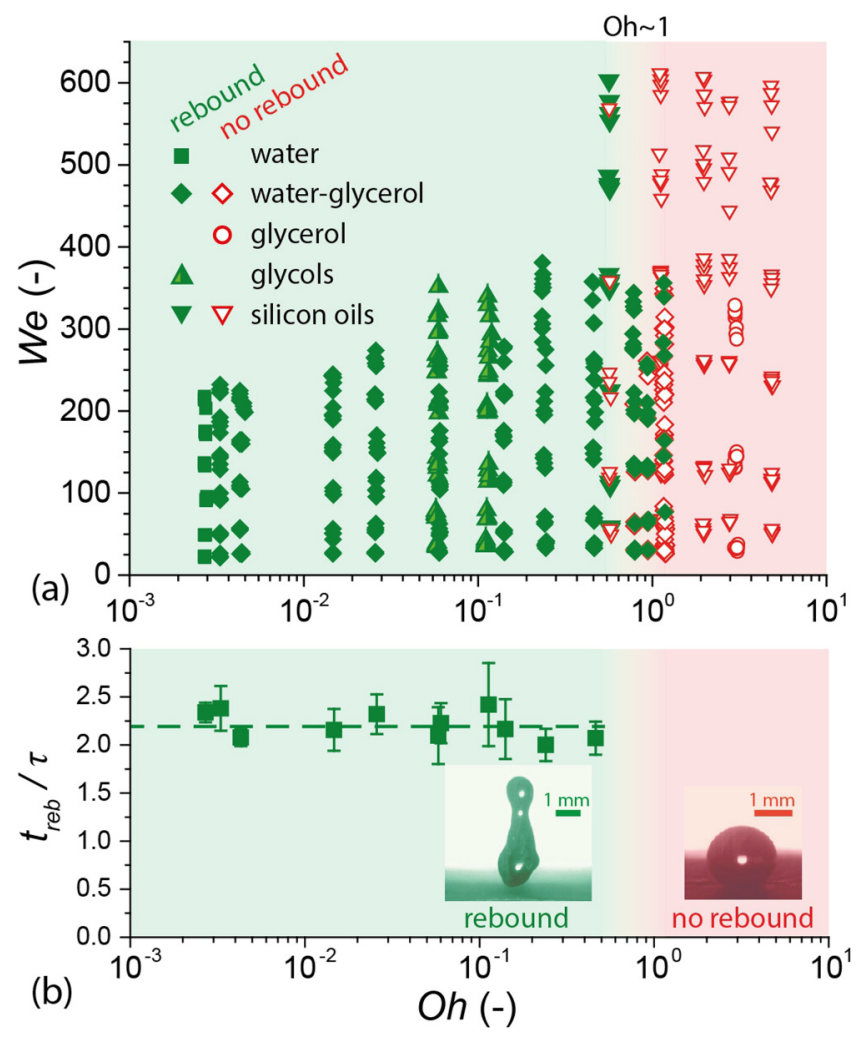

FIG. 2. (a) Rebound-no-rebound map for normal drop impact on a horizontal sublimating surface (603 impact tests with different liquids; see the legend). (b) Nondimensional rebound time $t_{\mathrm{reb}} / \tau$ as a function of the Ohnesorge number Oh.

on the substrate at $V_{\text {slide }} \approx V_{T}$. A linear scaling $V_{\text {slide }}=k V_{T}$ holds for all tested liquids, with $k$ depending on the Ohnesorge number [see Fig. 3(b)]. For a millimetric water drop $\left(\mathrm{Oh}=2.7 \times 10^{-3}\right)$, $k=0.9$, remarkably close to the ideal inviscid case $k=1$ and threefold higher than for water drops impacting on a superhydrophobic surface $(k=0.3$, as reported previously [42]). The enhanced sliding on a sublimating surface, even with respect to a superhydrophobic surface, is a consequence of the contactless regime, in which lateral adhesion forces are absent. The value of $k$ decreases with increasing Ohnesorge number [see Fig. 3(b)] and subsequently plateaus, remaining approximately constant at the value of $k \approx 0.6$ for $\mathrm{Oh}>1$, as shown by experiments. Values of $k<1$ denote that frictional losses in the vapor layer trapped between the drop and the substrate, despite being smaller than on superhydrophobic surfaces, are not negligible. As confirmed by the entropic lattice Boltzmann method, viscous losses occur mainly in the first (spreading) phase of the impact, over a time scale $t_{\mathrm{fr}}$, as a result of the drop rapid deformation and wall friction. Thus, the friction force can be estimated as $F_{\mathrm{fr}} \approx m\left(V_{T}-V_{\text {slide }}\right) / t_{\mathrm{fr}}=m V_{T}(1-k) / t_{\mathrm{fr}}$, which we will use below to estimate the drop angular velocity during tumbling. A thorough understanding of the dependence of $k$ on the Ohnesorge number, beyond the clear trend shown by the experimental data, would require the accurate reconstruction of the vapor layer flow at the liquid-solid interface through numerical simulations, a challenge that goes beyond the goals of the present study, but certainly provides motivation for future work.

Figure 4(a) shows the variation of the rebound time as a function of the tangential velocity for water and glycerol. Unsurprisingly, for water $\left(\mathrm{Oh}=2.7 \times 10^{-3}\right)$, the rebound time remains constant for a wide range of tangential impact velocity, up to $V_{T} \approx 2 \mathrm{~m} / \mathrm{s}$, and $t_{\mathrm{reb}} / \tau=2.2$ still holds, meaning 

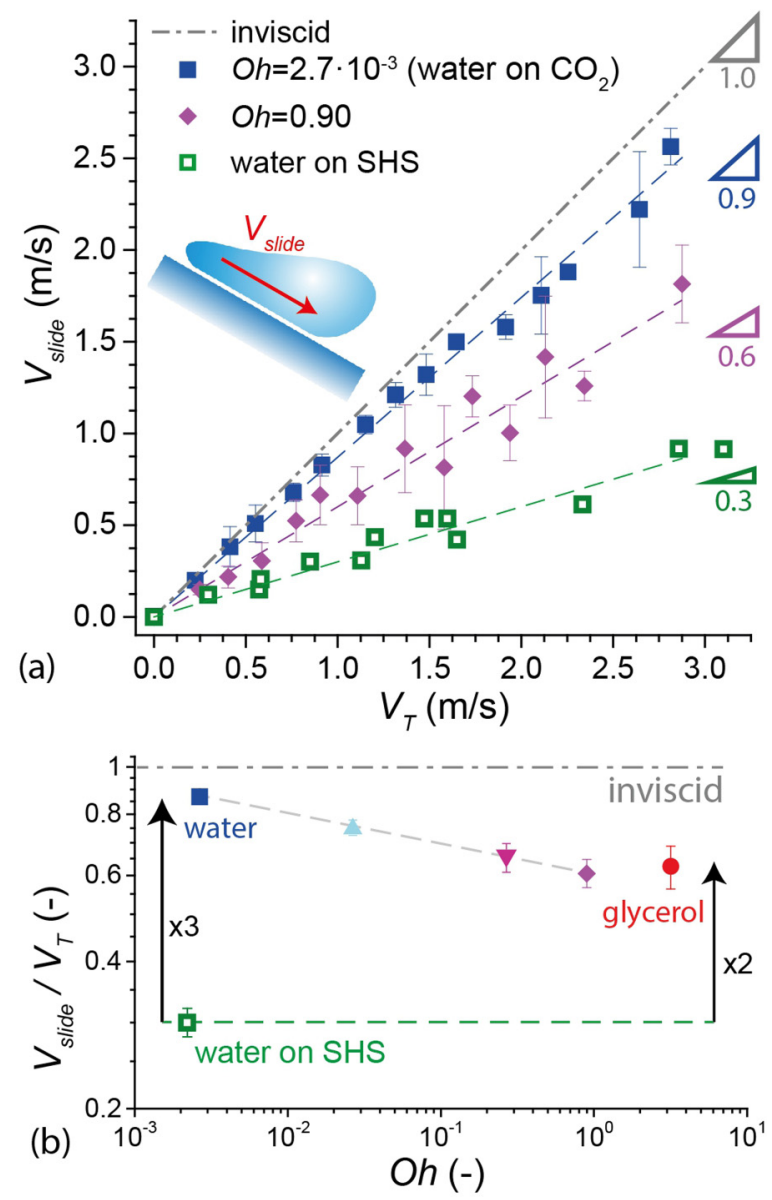

FIG. 3. (a) Drop average sliding velocity $V_{\text {slide }}$ as a function of impact tangential velocity $V_{T} ; V_{\text {slide }}$ is calculated as the ratio $L_{\text {slide }} / t_{\text {reb }}$. The legend reports values of the corresponding nondimensional Ohnesorge number Oh. (b) Ratio $k=V_{\text {slide }} / V_{T}$ as a function of Oh. The symbols denote the experimental data for impacts on the sublimating substrate: $\mathbf{\square}$, water; $\boldsymbol{\Delta}$, water-glycerol mixture 40:60; $\mathbf{\nabla}$, water-glycerol mixture 15:85; ๑, water-glycerol mixture 7:93; •, glycerol; and $\square$, water drop impact on a superhydrophobic surface (SHS) (data from [42]).

that the spreading and recoiling process of the drop is not affected by the simultaneous downward sliding. In other words, the usual picture of the inertia-capillarity interplay during the conventional rebound still holds and the viscosity (if low) does not play a prominent role. Only for $V_{T}>2 \mathrm{~m} / \mathrm{s}$, the impact deviates from the classical axisymmetric behavior: As demonstrated recently by Bird et al. [20] in the context of engineered superhydrophobic surfaces, the nonaxisymmetric spreading and recoiling (see video 7 in the SM [30]) can lead to a reduction of drop rebound time down to $t_{\mathrm{reb}} / \tau \approx 1.5$ for the maximum tested tangential velocity $\left(V_{T} \approx 3 \mathrm{~m} / \mathrm{s}\right)$.

However, for highly viscous glycerol drops $(\mathrm{Oh}=3.2)$ a fundamentally different rebound mechanism is identified, as highlighted previously in Fig. 1(c). The drop rebound starts at $V_{T} \approx 0.6 \mathrm{~m} / \mathrm{s}$ with a rebound time of $t_{\mathrm{reb}} / \tau \approx 2.2$ [see Fig. 4(a)], similar to that of water at the same $V_{T}$, and is significantly reduces down to a minimum of $t_{\mathrm{reb}} / \tau \approx 0.7$ at the highest tested tangential velocity $V_{T}=3 \mathrm{~m} / \mathrm{s}$. The glycerol rebound time is thus half that of water above $V_{T}=3 \mathrm{~m} / \mathrm{s}$, despite the fact that glycerol drops do not rebound for $V_{T}<0.6 \mathrm{~m} / \mathrm{s}$ and even with the three orders of magnitude higher viscosity of glycerol compared to water [Fig. 4(a)]. 


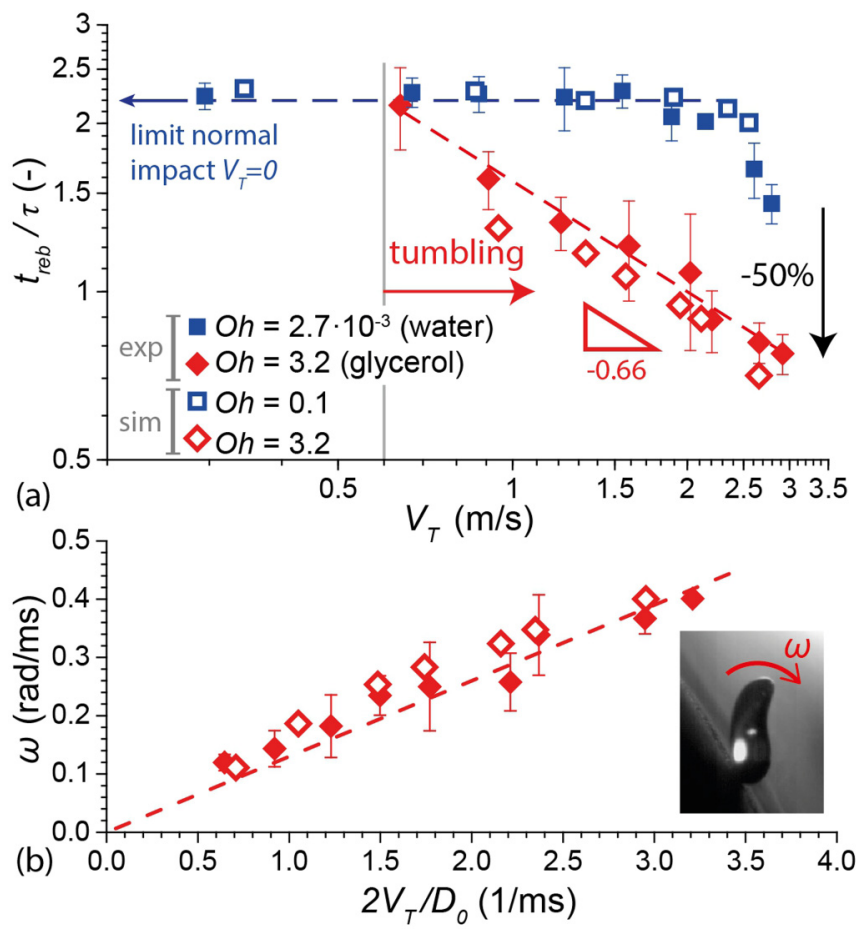

FIG. 4. (a) Nondimensional rebound time $t_{\mathrm{reb}} / \tau$ as a function of the tangential velocity $V_{T}$ : both experimental data for water and glycerol and numerical results are included. The legend reports values of Oh. (b) Angular velocity $\omega$ of highly viscous glycerol drop scales linearly with $2 V_{T} / D_{0}$. Closed symbols show the results of experiments and open symbols simulations. The fitting line is $\omega=0.13\left(2 V_{T} / D_{0}\right)$.

The reason for faster rebound at high viscosity is that, after some spreading and limited sliding, the drop tumbles off without recoiling. The drop rapidly detaches from the surface by rotating almost as a rigid body. The transition to a different rebound regime at high viscosities can be understood by comparing the drop relaxation time $t_{\text {rel }} \propto \mu D_{0} / 2 \sigma$ [43] to the characteristic oscillation time $\tau$, whose ratio is proportional to Oh. Indeed, at high viscosities, when $t_{\text {rel }} / \tau \sim \mathrm{Oh}>1$, the longer $t_{\text {rel }}$ delays drop recoiling and leads to sustaining the drop rotational energy during tumbling. Also, the distance traveled by the drop through sliding $L_{\text {slide }}=V_{\text {slide }} t_{\text {reb }}$ is significantly reduced for high Oh, since both $V_{\text {slide }}$ and $t_{\text {reb }}$ are reduced with increasing Oh, as shown in Figs. 3 and 4, respectively. Figure 4(b) shows that the average drop angular (spinning) velocity $\omega$ scales linearly with the ratio $2 V_{T} / D_{0}$ (see Sec. SM5 in the SM [30] for more details). The average angular velocity $\omega$ was defined and measured as the ratio of the angle formed by the major axis of the flattened drop and the substrate at the moment of drop lift-off $\left(\Omega_{\mathrm{reb}}\right)$, and the rebound time $t_{\text {reb }}$. Indeed, since the balance of the drop angular momentum gives $F_{\mathrm{fr}} D_{0} / 2 t_{\mathrm{fr}} \approx I \omega / t_{\mathrm{fr}}$, on the basis of the above estimation for $F_{\mathrm{fr}}$ we obtain $\omega \approx(1-k)\left(2 V_{T} / D_{0}\right)$, confirming the linear correlation. Figure 5 shows the value of $\Omega_{\text {reb }}$ as a function of the impact tangential velocity $V_{T} ; \Omega_{\text {reb }}$ is practically constant in the range $\sim 60^{\circ}-70^{\circ}$ for $V_{T}>1.3 \mathrm{~m} / \mathrm{s}$. As such, an increase of angular velocity $\omega$, corresponding to a faster spinning, is responsible for a significant reduction of the rebound time $t_{\text {reb }}$ for very viscous liquids with $\mathrm{Oh}>1$ (glycerol, $\mathrm{Oh}=3.2$ ) compared to the three orders of magnitude less viscous water.

A comparison of the drop shape evolution resulting from ELBM simulations between the lowviscosity regime $\mathrm{Oh}=0.1$ [see Fig. 6(a) and corresponding video 8 in the SM [30]] and the high-viscosity regime $\mathrm{Oh}=3.2$ [see Fig. 6(b)] highlights the same trend observed by experiments, 


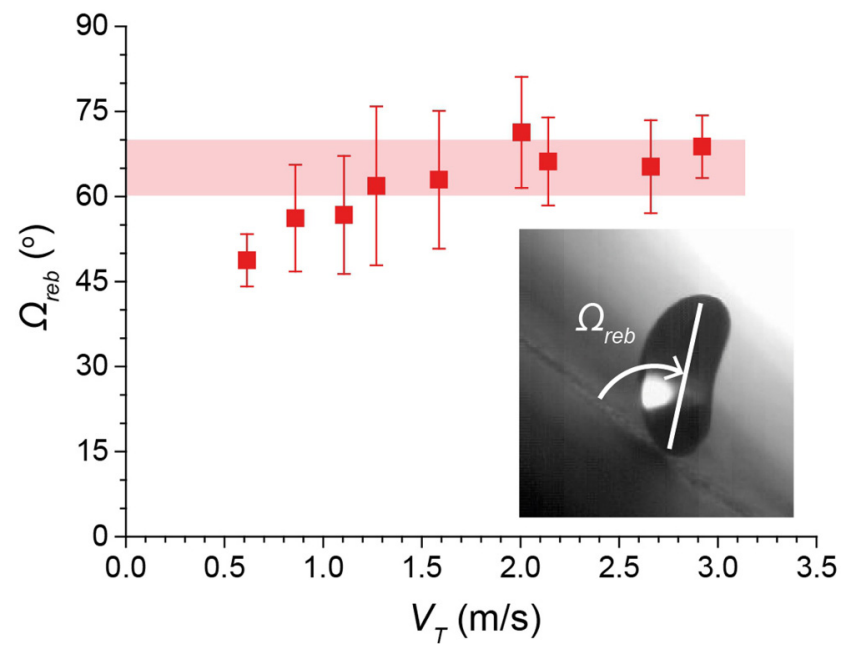

FIG. 5. Values of the drop tilting angle at the moment of rebound $\Omega_{\text {reb }}$ as a function of the tangential component of velocity $V_{T}$ for a highly viscous glycerol drop $(\mathrm{Oh}=3.2)$.

confirming the limited role of viscosity on drop dynamics in the regime $\mathrm{Oh}<1$ and the occurrence of tumbling rebound for $\mathrm{Oh}>1$.

The occurrence of tumbling can be better understood within the framework of vorticity generation from a boundary (shear) layer. Injection of vorticity through a shear layer was studied, in particular by [44], in a direct numerical simulation of a drop normal impact onto a flat surface. Transition to tumbling under shear is a common scenario also away from boundaries [45]. In the present experiment, the tilted slope provides an off-center impact condition and thus can cause generation of the angular momentum. Hence, when a viscous drop impacts the sublimating surface, the linear momentum of the drop is partially converted into angular momentum, providing the drop with a
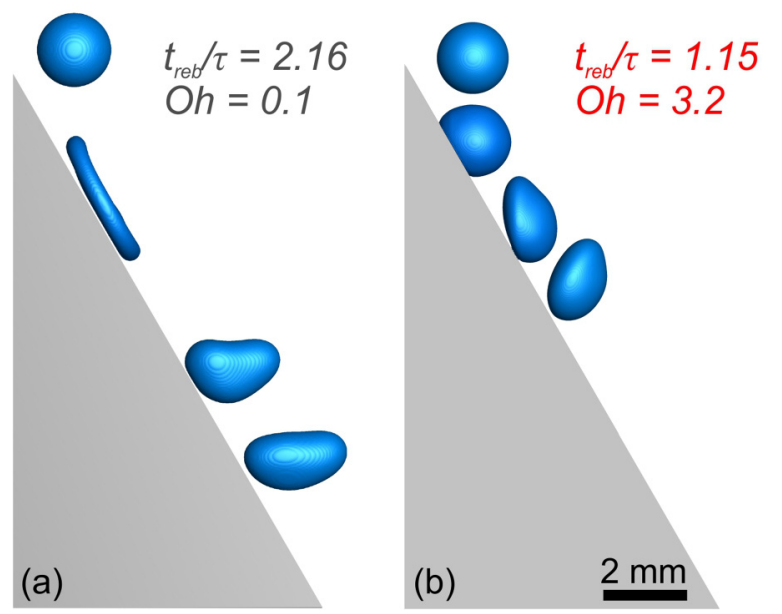

FIG. 6. Evolution of simulated drop impacting on a sublimating slope: (a) a drop in the low-viscosity regime $\left(\mathrm{Oh}=0.1, \mathrm{We}=121\right.$, and $\left.\alpha=60^{\circ}\right)$ and (b) a drop in the high-viscosity regime $(\mathrm{Oh}=3.2, \mathrm{We}=121$, and $\alpha=60^{\circ}$ ). The rebound time in the simulation was identified through matching the experimentally observed orientation of the drop at the rebound with the corresponding simulated image. 


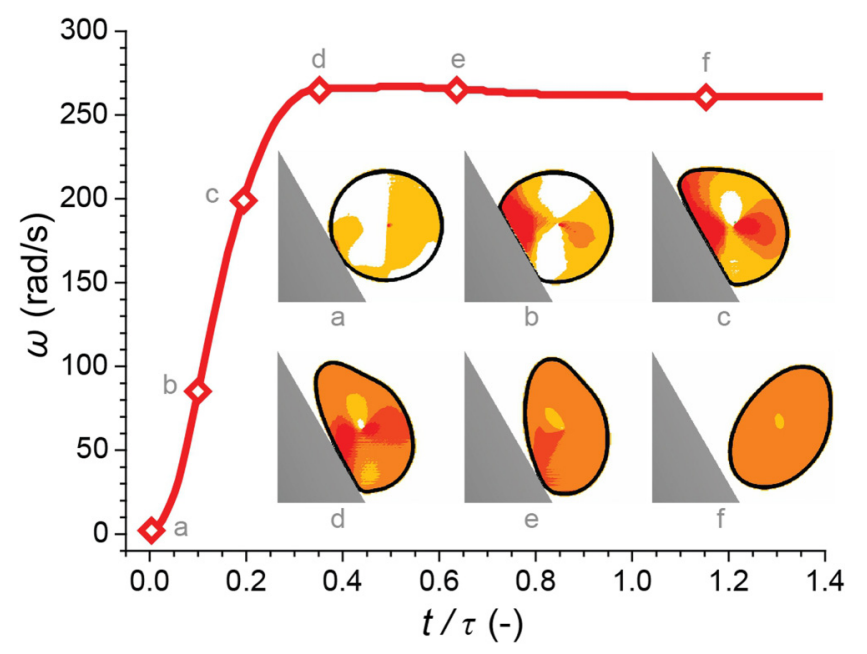

FIG. 7. History of the average angular velocity $\omega$ of highly viscous liquid drop with $\mathrm{Oh}=3.2$, We $=121$, and $\alpha=60^{\circ}$ during tumbling (simulation). Propagation of the dominant angular velocity component of $\vec{\omega}_{\text {loc }}$ inside the drop is shown in the insets (a-f), at different stages of tumbling: stages a-c show the initial rise of the angular velocity shortly after the impact and stages $\mathrm{d}-\mathrm{f}$ show rotation of the drop acting essentially as a solid. The drop rebounds (stage f) at $t / \tau=1.15$. A shading or color increase corresponds to the increase of the clockwise rotation.

spin that facilitates takeoff causing tumbling. Note that the energy associated with the rotation $R \sim m D_{0}^{2} \omega^{2} / 8$ is small compared to the tangential component of translational kinetic energy $K \sim m V_{T}^{2} / 2$. Since $\omega=0.13\left(2 V_{T} / D_{0}\right)$ [Fig. 4(b)], then $R / K \sim 10^{-2}$; that is, only about $1 \%$ of the drop initial kinetic energy is converted into rotational energy.

\section{Vorticity generation and evolution}

To better identify the origin of the tumbling effect, the local angular velocity was calculated from the numerical simulations as $\vec{\omega}_{\text {loc }}=|\vec{r} \times \vec{v}| / r^{2}$, where $\vec{r}$ is the position vector relative to the center-of-mass of the drop and $\vec{v}$ is the relative fluid velocity at that location. The component of the vector $\vec{\omega}_{\text {loc }}$, orthogonal to the plane of symmetry of the impacting drop, is overwhelmingly dominant, i.e., two orders of magnitude larger than the other two orthogonal components, since the rotation axis is essentially perpendicular to the plane of symmetry. From this dominant component, the average instantaneous angular velocity over the drop volume $\omega(t)$ was computed: Its evolution is illustrated in Fig. 7 (see also the corresponding video 9 in [30]) together with the characteristic snapshots of the local angular velocity spatial distribution on the symmetry plane; $\omega$ rapidly reaches a maximum value well before rebounding, with the rotational motion initiated near the impact zone and then becoming uniform. Thereafter, $\omega$ remains practically constant through the liquid, with the drop exhibiting the behavior of a solid ready to tumble. Put differently, the oblique impact breaks the axisymmetry of the drop at the beginning of the sliding and the gain in angular momentum is thus initiated by the off-center flow reversal. If the relaxation time is large enough $(\mathrm{Oh}>1)$, the angular momentum diffuses through the entire drop before it can recoil and the tumbling takes place.

For comparison, Fig. 8 shows the history of the averaged angular velocity $\omega$ in the low-viscosity regime $(\mathrm{Oh}=0.1)$ together with the characteristic snapshots of the distribution of the local angular velocity during impact (see also video 10 in [30]). Unlike in the case of high viscosity, the angular velocity gained initially in the impact (phases a-c in Fig. 8) is not sustained at a constant value during the extension and sliding (phases $\mathrm{c}$ and $\mathrm{d}$ ) and no solidlike tumbling is observed. At the final stage close to the rebound (phase $\mathrm{f}$ ), a small gain of the average angular velocity is due to the merging 


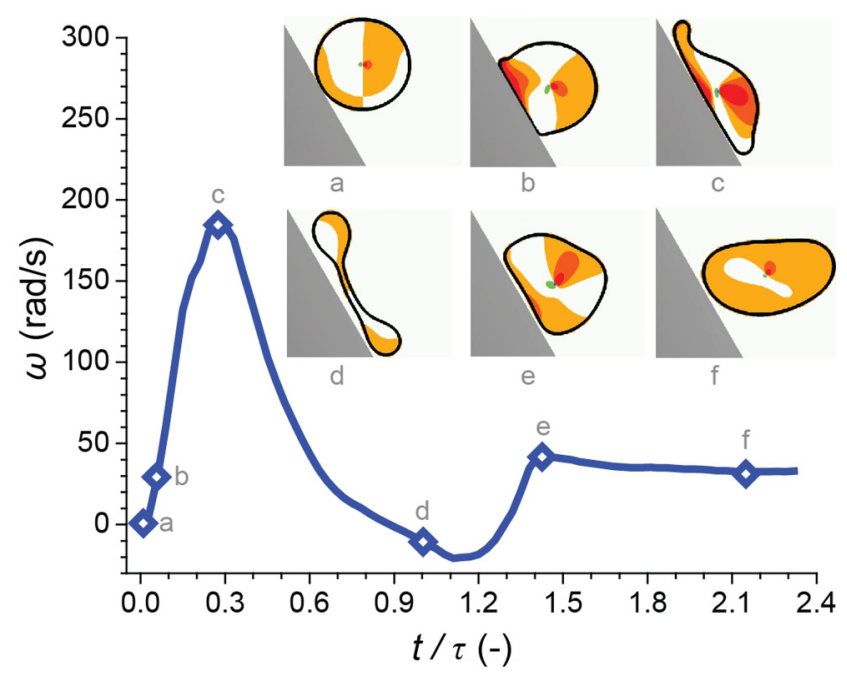

FIG. 8. History of the average angular velocity $\omega$ of a low-viscosity liquid drop with $\mathrm{Oh}=0.1, \mathrm{We}=121$, and slope $\alpha=60^{\circ}$ (simulation). Propagation of the dominant angular velocity component of $\vec{\omega}_{\text {loc }}$ inside the drop is shown in the insets (a-f), at different stages of drop impact. The drop rebounds (stage $\mathrm{f}$ ) at $t / \tau=2.1$. A shading or color increase corresponds to the increase of the clockwise rotation.

of the dumbbell-like shape of the drop at the intermediate stage, with nonaxisymmetric collapse of the rim from phase d to e. In other words, in the low-viscosity regime the fluid slips away and the rotation is not sustained.

\section{CONCLUSIONS}

Taken together, our results demonstrate and explain the existence of a prompt tumbling rebound regime for nonaxisymmetric drop impact on surfaces under slip conditions, here readily realized with the help of a sublimating slope. To this end, sublimating surfaces present themselves as an interesting, easy to use platform for the study of unexplored, liquid-surface interactions, especially in the limit of small friction, brought about by the absence of direct contact between the liquid and the solid surface. Similar effects could be possible also on superhydrophobic surfaces, if they are fabricated to reach extreme performance. Preliminary simulations for contact angles $170^{\circ}<\theta<180^{\circ}$ indicate that tumbling rebound could take place, opening an interesting direction for future work, e.g., to promote repellence of viscous supercooled drops in icing conditions $[31,46]$.

\section{ACKNOWLEDGMENTS}

C.A. acknowledges funding through a Marie Curie Intra-European Fellowship (Grant No. 301174). I.K. and S.S.C. were supported by the ERC Grant No. 291094-ELBM. A.M.M. was supported by the ETH Grant No. ETH35-12-2. Computational resources at CSCS, Switzerland, were provided under the Grant No. S492. The authors acknowledge Marti Ueli (ETH Zurich) for technical support in the liquid viscosity measurement.

[1] I. V. Roisman, E. Berberović, and C. Tropea, Inertia dominated drop collisions. I. On the universal flow in the lamella, Phys. Fluids 21, 052103 (2009). 
[2] I. V. Roisman, Inertia dominated drop collisions. II. An analytical solution of the Navier-Stokes equations for a spreading viscous film, Phys. Fluids 21, 052104 (2009).

[3] A. L. Yarin, Drop impact dynamics: Splashing, spreading, receding, bouncing, Annu. Rev. Fluid Mech. 38, 159 (2006).

[4] M. Marengo, C. Antonini, I. V. Roisman, and C. Tropea, Drop collisions with simple and complex surfaces, Curr. Opin. Colloid Interface Sci. 16, 292 (2011).

[5] D. Bonn, J. Eggers, J. Indekeu, J. Meunier, and E. Rolley, Wetting and spreading, Rev. Mod. Phys. 81, 739 (2009).

[6] M. Mani, S. Mandre, and M. P. Brenner, Events before droplet splashing on a solid surface, J. Fluid Mech. 647, 163 (2010).

[7] S. Mandre and M. P. Brenner, The mechanism of a splash on a dry solid surface, J. Fluid Mech. 690, 148 (2011).

[8] G. Riboux and J. M. Gordillo, Experiments of Drops Impacting a Smooth Solid Surface: A Model of the Critical Impact Speed for Drop Splashing, Phys. Rev. Lett. 113, 024507 (2014).

[9] L. Xu, Liquid drop splashing on smooth, rough, and textured surfaces, Phys. Rev. E 75, 056316 (2007).

[10] L. Xu, W. Zhang, and S. Nagel, Drop Splashing on a Dry Smooth Surface, Phys. Rev. Lett. 94, 184505 (2005).

[11] C. Antonini, I. Bernagozzi, S. Jung, D. Poulikakos, and M. Marengo, Water Drops Dancing on Ice: How Sublimation Leads to Drop Rebound, Phys. Rev. Lett. 111, 014501 (2013).

[12] L. H. J. Wachters and N. A. J. Westerling, The heat transfer from a hot wall to impinging water drops in the spheroidal state, Chem. Eng. Sci. 21, 1047 (1966).

[13] A. L. Biance, C. Clanet, and D. Quéré, Leidenfrost drops, Phys. Fluids 15, 1632 (2003).

[14] T. Tran, H. J. J. Staat, A. Prosperetti, C. Sun, and D. Lohse, Drop Impact On Superheated Surfaces, Phys. Rev. Lett. 108, 036101 (2012).

[15] T. Tran, H. J. J. Staat, A. Susarrey-Arce, T. C. Foertsch, A. van Houselt, H. J. G. E. Gardeniers, A. Prosperetti, D. Lohse, and C. Sun, Droplet impact on superheated micro-structured surfaces, Soft Matter 9, $3272(2013)$.

[16] J. M. Kolinski, S. M. Rubinstein, S. Mandre, M. P. Brenner, D. A. Weitz, and L. Mahadevan, Skating on a Film of Air: Drops Impacting on a Surface, Phys. Rev. Lett. 108, 074503 (2012).

[17] J. M. Kolinski, L. Mahadevan, and S. M. Rubinstein, Lift-Off Instability During the Impact of a Drop on a Solid Surface, Phys. Rev. Lett. 112, 134501 (2014).

[18] J. de Ruiter, R. Lagraauw, D. van den Ende, and F. Mugele, Wettability-independent bouncing on flat surfaces mediated by thin air films, Nat. Phys. 11, 48 (2015).

[19] D. Richard, C. Clanet, and D. Quéré, Contact time of a bouncing drop, Nature (London) 417, 811 (2002).

[20] J. C. Bird, R. Dhiman, H.-M. Kwon, and K. K. Varanasi, Reducing the contact time of a bouncing drop, Nature (London) 503, 385 (2013).

[21] Y. Liu, L. Moevius, X. Xu, T. Qian, J. M. Yeomans, and Z. Wang, Pancake bouncing on superhydrophobic surfaces, Nat. Phys. 10, 515 (2014).

[22] C. Antonini, F. Villa, I. Bernagozzi, A. Amirfazli, and M. Marengo, Drop rebound after impact: The role of the receding contact angle, Langmuir 29, 16045 (2013).

[23] M. A. Goldshtik, V. M. Khanin, and V. G. Ligai, A liquid drop on an air cushion as an analogue of Leidenfrost boiling, J. Fluid Mech. 166, 1 (2006).

[24] J. Snoeijer, P. Brunet, and J. Eggers, Maximum size of drops levitated by an air cushion, Phys. Rev. E 79, 036307 (2009).

[25] P. Brunet and J. H. Snoeijer, Star-drops formed by periodic excitation and on an air cushion - A short review, Eur. Phys. J. Spec. Top. 192, 207 (2011).

[26] G. Lagubeau, M. Le Merrer, C. Clanet, and D. Quéré, Leidenfrost on a ratchet, Nat. Phys. 7, 395 (2011).

[27] D. Quéré, Leidenfrost dynamics, Annu. Rev. Fluid Mech. 45, 197 (2013).

[28] I. U. Vakarelski, N. A. Patankar, J. O. Marston, D. Y. C. Chan, and S. T. Thoroddsen, Stabilization of Leidenfrost vapour layer by textured superhydrophobic surfaces, Nature (London) 489, 274 (2012).

[29] H.-J. Butt, C. Semprebon, P. Papadopoulos, D. Vollmer, M. Brinkmann, and M. Ciccotti, Design principles for superamphiphobic surfaces, Soft Matter 9, 418 (2013). 


\section{CONTACTLESS PROMPT TUMBLING REBOUND OF DROPS ...}

[30] See Supplemental Material at http://link.aps.org/supplemental/10.1103/PhysRevFluids.1.013903 for movies and additional experimental details.

[31] T. Maitra, C. Antonini, M. K. Tiwari, A. Mularczyk, Z. Imeri, P. Schoch, and D. Poulikakos, Supercooled water drops impacting superhydrophobic textures, Langmuir 30, 10855 (2014).

[32] R. Rioboo, M. Voué, A. Vaillant, and J. De Coninck, Drop impact on porous superhydrophobic polymer surfaces, Langmuir 24, 14074 (2008).

[33] L. F. Loucks, Subtleties of phenomena involving ice-water equilibria, J. Chem. Educ. 63, 115 (1986).

[34] A. Mazloomi, S. S. Chikatamarla, and I. V. Karlin, Entropic Lattice Boltzmann Method for Multiphase Flows, Phys. Rev. Lett. 114, 174502 (2015).

[35] I. V. Karlin, A. N. Gorban, S. Succi, and V. Boffi, Maximum Entropy Principle for Lattice Kinetic Equations, Phys. Rev. Lett. 81, 6 (1998).

[36] S. S. Chikatamarla, S. Ansumali, and I. V. Karlin, Entropic Lattice Boltzmann Models for Hydrodynamics in Three Dimensions, Phys. Rev. Lett. 97, 010201 (2006).

[37] S. S. Chikatamarla and I. V. Karlin, Entropy and Galilean Invariance of Lattice Boltzmann Theories, Phys. Rev. Lett. 97, 190601 (2006).

[38] J. Zhang and D. Y. Kwok, Lattice Boltzmann study on the contact angle and contact line dynamics of liquid-vapor interfaces, Langmuir 20, 8137 (2004).

[39] A. Mazloomi M., S. S. Chikatamarla, and I. V. Karlin, Entropic lattice Boltzmann method for multiphase flows: Fluid-solid interfaces, Phys. Rev. E 92, 023308 (2015).

[40] E. Pierce, F. J. Carmona, and A. Amirfazli, Understanding of sliding and contact angle results in tilted plate experiments, Colloids Surf. A 323, 73 (2008).

[41] H. B. Eral, D. J. C. M.'t Mannetje, and J. M. Oh, Contact angle hysteresis: A review of fundamentals and applications, Colloid Polym. Sci. 291, 247 (2012).

[42] C. Antonini, F. Villa, and M. Marengo, Oblique impacts of water drops onto hydrophobic and superhydrophobic surfaces: Outcomes, timing, and rebound maps, Exp. Fluids 55, 1713 (2014).

[43] K. Moran, A. Yeung, and J. Masliyah, Shape relaxation of an elongated viscous drop, J. Colloid Interface Sci. 267, 483 (2003).

[44] R. D. Schroll, C. Josserand, S. Zaleski, and W. W. Zhang, Impact of a Viscous Liquid Drop, Phys. Rev. Lett. 104, 034504 (2010).

[45] J. M. Skotheim and T. W. Secomb, Red Blood Cells and Other Nonspherical Capsules in Shear Flow: Oscillatory Dynamics and the Tank-Treading-to-Tumbling Transition, Phys. Rev. Lett. 98, 078301 (2007).

[46] T. M. Schutzius, S. Jung, T. Maitra, P. Eberle, C. Antonini, C. Stamatopoulos, and D. Poulikakos, Physics of icing and rational design of surfaces with extraordinary icephobicity, Langmuir 31, 4807 (2015). 